\title{
Geometría, formalismo e intuición: David Hilbert y el método axiomático formal (1891-1905)
}

\author{
(Geometry, Formalism and Intuition: \\ David Hilbert and the formal Axiomatic \\ Method (1891-1905))
}

\author{
Eduardo N. GiOvanNINI
}

Recibido: 24 de mayo de 2013

Aceptado: 28 de enero de 2014

\section{Resumen}

El artículo presenta y analiza un conjunto de notas manuscritas de clases para cursos sobre geometría, dictados por David Hilbert entre 1891 y 1905. Se argumenta que en estos cursos el autor elabora la concepción de la geometría que subyace a sus investigaciones axiomáticas en Fundamentos de la geometría (1899). Por un lado, afirmo que lo que caracteriza esta concepción de la geometría es: i) una posición axiomática abstracta o formal; ii) una posición empirista respecto del origen de la geometría y de su lugar dentro de las distintas teorías matemáticas. Por otro lado, sostengo que el papel que Hilbert le confiere a la intuición geométrica en el proceso de axiomatización (formal) de esta teoría, permite apreciar claramente su oposición respecto de las posiciones formalistas (extremas) con las que habitualmente es identificado.

Palabras clave: Hilbert, método axiomático, geometría, formalismo, intuición.

\section{Abstract}

The paper presents and analyzes a set of unpublished notes for lecture courses on geometry, which David Hilbert gave between 1891 and 1905. It will be argued 
that in these lecture courses the author elaborates the conception of geometry which underlies his axiomatic investigations in Foundations of Geometry (1899). On the one hand, it is claimed that this conception of geometry can be characterized by: $i$ ) an abstract or formal axiomatic view; ii) an empiricist stance regarding the origins of geometry and its place among the different mathematical theories. On the other hand, it is asserted that the role that Hilbert assigns to geometrical intuition in the process of the (formal) axiomatization of this theory, can be used to perceive his clear opposition with regard to (radical) formalist views which he is often identified with.

Keywords: Hilbert, axiomatic method, geometry, formalism, intuition.

\section{Introducción}

La axiomatización de la geometría llevada a cabo por David Hilbert (18621943) en su monografía Fundamentos de la geometría (1899) suele ser considerada una de sus contribuciones más importantes a la matemática moderna. Esta relevancia no descansa exclusivamente en los resultados matemáticos alcanzados en este célebre trabajo, sino en gran medida en las ideas metodológicas o fundacionales allí elaboradas. El libro de Hilbert constituye una de las primeras instancias históricas, y quizás la más influyente, del método axiomático formal. Dicha presentación axiomática de la geometría euclídea trajo aparejada una nueva manera de entender la naturaleza de las teorías geométricas y matemáticas en general, que logró capturar magistralmente el creciente impulso hacia la abstracción y la sistematización que venía dominando la matemática desde la segunda mitad del siglo XIX. Sin embargo, en la medida en que esta nueva concepción abstracta o formal del método axiomático incluía una filosofía de la matemática, ésta no fue una filosofía que el propio Hilbert se ocupó de exponer, al menos en este periodo inicial y en textos publicados.

Ahora bien, la monografía de Hilbert publicada originalmente en 1899 no fue el resultado de un interés repentino del autor por el problema de los fundamentos de la geometría. Si bien en esta etapa temprana sus investigaciones matemáticas se centraron inicialmente en la teoría de invariantes, y luego en la teoría de números algebraicos ${ }^{1}$, sabemos que desde 1891 Hilbert impartió regularmente cursos universitarios sobre geometría. Afortunadamente, este material quedó registrado en forma de notas cuidadosamente elaboradas para cursos sobre geometría, dictados en las universidades de Königsberg y Göttingen. ${ }^{2}$

\footnotetext{
1 Sobre estos primeros trabajos de Hilbert puede verse Reid (1996) y Rowe (2000).

2 Las notas de clases de Hilbert para cursos sobre geometría han sido parcialmente publicadas en el primer volumen de la Hilbert Edition. Para una descripción general del carácter de estos manuscritos,
} 
Estas fuentes manuscritas han abierto una nueva perspectiva desde donde analizar las contribuciones de Hilbert a los fundamentos de la geometría y a la concepción abstracta del método axiomático. La notable importancia de estos manuscritos reside en que los diversos resultados matemáticos allí alcanzados son complementados con numerosas observaciones y reflexiones respecto de las implicancias metodológicas y filosóficas de su novedoso abordaje axiomático formal a la geometría. A diferencia de Fundamentos de la geometría (1899), en sus cursos Hilbert no se limita a aplicar el método axiomático formal a la geometría, sino que además realiza importantes consideraciones respecto de las consecuencias que su nueva concepción del método axiomático conlleva para la compresión de la naturaleza de la geometría y de la matemática en general.

El objetivo de este artículo es utilizar el material que aportan estas fuentes manuscritas para reexaminar el abordaje axiomático a la geometría llevado adelante por Hilbert en la primera etapa de sus trabajos sobre los fundamentos de la matemática, que se extiende aproximadamente entre 1891 y 1905.3 Sostendré que, en estas notas de clases, Hilbert elaboró y presentó la concepción axiomática de la geometría que subyace a su presentación de la geometría euclídea en su libro de 1899. Por concepción axiomática de la geometría no entenderé aquí una exposición de carácter sistemático, en el sentido de una filosofía de la geometría cuidadosamente elaborada y completamente articulada. Por el contrario, con ello aludiré más bien a una serie de reflexiones y observaciones, de tenor claramente filosófico, respecto de: $i$ ) la naturaleza de la geometría y del conocimiento geométrico en general; ii) el lugar que ocupa la geometría en el contexto de la matemática en general y cómo se relaciona esta disciplina con otras ramas matemáticas; iii) el papel que desempeña la intuición en las investigaciones geométricas, en particular en el proceso de axiomatización; iv) la naturaleza y función del método axiomático, en particular en su aplicación a la geometría. ${ }^{4}$ De este modo, intentaré mostrar que un estudio de estas fuentes permite ofrecer una interpretación mejor contextualizada e históricamente más adecuada de la concepción del método axiomático defendida por Hilbert en este período inicial de sus trabajos sobre los fundamentos de la mate-

como así también de este proyecto editorial, puede consultarse Toepell (1986) y Majer y Hallett (2004).

${ }^{3}$ En la literatura es habitual distinguir dos etapas principales en los trabajos de Hilbert sobre los fundamentos de la matemática. La primera etapa, designada "geométrica", se desarrolla aproximadamente de 1891 hasta 1905 y tiene a la articulación de la concepción formal o abstracta del método axiomático como su desarrollo teórico más notable. En cambio, la segunda etapa, que tiene lugar entre 1917 y 1931, se concentra principalmente en el problema de la consistencia de la aritmética y el análisis. A esta segunda etapa corresponde el famoso "programa de Hilbert", cuyo objetivo central era presentar una prueba de la consistencia de la aritmética en la que se utilicen estrictamente métodos 'finitarios" de demostración. La creación de la "teoría de la demostración" [Beweistheorie] se reconoce como la contribución más importante de esta segunda "etapa aritmética".

${ }^{4}$ Este carácter no sistemático de las reflexiones de Hilbert ha sido enfatizado por Corry (2006). 
mática, cuyo punto culminante fue la publicación de su monografía Fundamentos de la geometría (1899).

El artículo se organizará de la siguiente manera: en la primera sección presentaré la concepción de la geometría exhibida por Hilbert en estas notas de clases. Seguidamente, examinaré cómo es caracterizada en estas fuentes la nueva concepción abstracta del método axiomático. Finalmente, ello me llevará en las dos secciones siguientes a analizar una de las cuestiones más enfatizadas a lo largo de estos cursos, que identificaré como uno de los aspectos más llamativos de la temprana concepción axiomática de la geometría de Hilbert, a saber: el modo en que concibe y explica la relación entre la intuición geométrica, señalada como una de las fuentes primarias de conocimiento geométrico, y el nuevo método axiomático formal.

\section{La temprana concepción de la geometría}

La primera incursión de Hilbert en el campo de la geometría consistió en un conjunto de notas de clases para un curso sobre geometría proyectiva, dictado en Königsberg en el semestre de verano de 1891.5 En este primer curso se aprecia claramente que la presentación axiomática de Hilbert se construyó sobre la base de la tradición de la geometría sintética, que tomó un renovado impulso a partir de los trabajos de Gaspard Monge (1746-1818), Victor Poncelet (1788-1867), Karl G. C. von Staudt (1798-1867) y Jakob Steiner (1796-1863).6 Especialmente importante para esta tradición era evitar donde fuera posible la introducción de consideraciones numéricas en la construcción de las teorías geométricas. De este modo, encontramos en estas notas de clases numerosas referencias elogiosas a von Staudt, quien fue el primero en hacer de la geometría proyectiva una ciencia autónoma, "en la que no se mide ni se calcula, sino que sólo se construye" (Hilbert 1891, p. 25).

Por otro lado, Hilbert enuncia en la introducción de este primer curso una tesis filosófica fundamental respecto de la naturaleza de las teorías matemáticas en general. Esta tesis constituía una posición muy influyente y diseminada en el siglo XIX, especialmente entre los matemáticos alemanes, y fue quizás Gauss el primero en formularla de un modo explícito:

Según mi más profundo convencimiento, la teoría del espacio tiene en nuestro conocimiento a priori un lugar completamente distinto que la pura teoría de las magnitudes [reine Grössenlehre]; nuestro conocimiento de la primera carece de aquel completo convencimiento de su necesidad (y también de su verdad) que es propio de la segunda.

\footnotetext{
${ }^{5}$ Cf. Hilbert (1891).

6 Toepell (1986) ha analizado detalladamente las fuentes utilizadas por Hilbert en este período previo a Fundamentos de la geometría (1899).
} 
Debemos humildemente admitir que, mientras el número es sólo un producto de nuestro pensamiento, el espacio tiene además una realidad fuera de nuestro pensamiento, a la cual no podemos prescribirle a priori sus leyes. (Gauss a Besell, 9 de abril de 1830; en Gauss y Bessel (1880), p. 497)7

De acuerdo con esta tesis de Gauss, compartida por Riemann y H. Grassmann entre otros matemáticos notables, en matemática se debe distinguir entre aquellas disciplinas que pueden ser fundadas exclusivamente en el pensamiento puro y aquellas que, al menos en parte, tienen su origen en la experiencia. En virtud de su origen epistemológico, es preciso distinguir entre la matemática pura (aritmética, álgebra, análisis, teoría de números, etc.) y la matemática mixta ${ }^{8}$, en donde se encuentran la geometría y la mecánica. Hilbert reproduce esta distinción en un pasaje muy elocuente del curso sobre geometría proyectiva de 1891:

La geometría es la ciencia de las propiedades del espacio, y se diferencia substancialmente de las ramas matemáticas puras, como la teoría de números, el álgebra y la teoría de funciones. Los resultados de estas disciplinas pueden ser alcanzados a través del pensamiento puro [...] Algo completamente distinto ocurre en la geometría. No puedo nunca fundar las propiedades del espacio en la mera reflexión, tanto como no puedo reconocer de ese modo las leyes básicas de la mecánica, las leyes de la gravitación o cualquier otra ley física. El espacio no es un producto de mi pensamiento, sino que me es dado sólo a través de los sentidos [Sinne]. Necesito de la intuición y el experimento, tanto como se los requiere para fundar las leyes físicas, donde también la materia debe sernos dada a través de los sentidos. (Hilbert 1891, pp. 22-23)

En la medida en que en sus trabajos posteriores Hilbert adopte una posición axiomática abstracta, esta definición tradicional de la geometría como la ciencia que se ocupa de estudiar las propiedades del espacio (físico) deberá ser abandonada. Sin embargo, una característica central de su concepción de la geometría que aparece sugerida aquí tempranamente, y que se mantendrá a lo largo de todo este período inicial, es una postura empirista respecto del estatus epistemológico de esta teoría matemática. Es decir, Hilbert sostiene que el conjunto de "hechos geométricos fundamentales" sobre los que se construye nuestro conocimiento geométrico proviene de la experiencia, y por lo tanto tiene un carácter empírico:

Entre los fenómenos o hechos de la experiencia que se nos ofrecen en la observación de la naturaleza, existe un grupo particularmente destacado, es decir, el grupo de aquellos hechos que determinan la forma externa de las cosas [die äussere Gestalt der Dinge]. De estos hechos se ocupa la geometría. (Hilbert 1893/1894, p.72)

\footnotetext{
7 Para un análisis de la distinción gaussiana entre geometría y aritmética, y su influencia en la tradición matemática alemana del siglo XIX, véase Ferreirós (2006).

8 Adopto esta expresión de Ferreirós (2006).
} 
La posición empirista de Hilbert consiste en afirmar que la geometría se funda en un conjunto de hechos, leyes y conceptos básicos que no pueden ser adquiridos a través del pensamiento puro, sino que nos son dados a través de la experiencia y la intuición. El trabajo del geómetra consiste precisamente describir y ordenar este conjunto de hechos, del mismo modo que la tarea del físico consiste en describir y ordenar un determinado conjunto de fenómenos físicos. ${ }^{9}$ Asimismo, al indicar que la geometría es la ciencia encargada de estudiar el grupo de hechos que determina la forma externa de las cosas en el espacio, Hilbert reconoce que las proposiciones básicas de la geometría elemental no son muy distintas que las proposiciones de la física en cuanto a que, en un sentido factual, formulan una multitud de hechos del "mundo exterior" [Aussenwelt]. Hilbert subraya de ese modo el papel significativo de la geometría en nuestro conocimiento de la naturaleza, en tanto puede ser utilizada para suministrar una descripción más o menos precisa de la naturaleza. ${ }^{10} \mathrm{En}$ este aspecto, la geometría elemental puede ser considerada así una de las primeras ramas de la fisica.

Hilbert advierte además que la geometría se diferencia de otras teorías físicas como la mecánica, la teoría de la electricidad, la óptica, etc., no en virtud de una característica esencial asociada a su naturaleza, sino más bien debido a su avanzado estado de desarrollo. El notable grado de avance que ha alcanzado la geometría desde los tiempos de Euclides y el consenso generalizado respecto de los 'hechos' que forman este dominio o ámbito de conocimiento permiten, según Hilbert, que esta disciplina pueda ser sometida fácilmente a un tratamiento axiomático (formal). ${ }^{11}$ En este preciso sentido, la geometría puede ser considerada la más completa de las ciencias naturales:

La geometría elemental (euclídea) tiene como objeto los hechos y leyes que el comportamiento [Verhalten] espacial de las cosas nos presenta. Según su estructura, es un sistema de proposiciones [Sätzen] que - en mayor o menor medida - pueden ser deducidas de un modo puramente lógico a partir de ciertas proposiciones indemostrables, los axiomas. Esta conducta, que en menor completitud encontramos, por ejemplo, en la física matemática, puede expresarse brevemente en la sentencia: la geometría es la ciencia natural más completa. (Hilbert 1898/1899, p. 302)

Hilbert adopta en su concepción temprana de la geometría una posición visiblemente empirista, que en cierta medida puede resultar insospechada para aquellos más familiarizados con su exposición axiomática formal de la geometría en Fundamentos de la geometría (1899). Ahora bien, esta posición empirista no es pro-

\footnotetext{
9 Véase Hilbert (1893/1894), p. 72.

10 Cf. Hilbert (1898/1899a), p. 221.

11 Cf. Hilbert (1893/1894), p. 72.
} 
fundizada en ningún momento, sino que se circunscribe a defender que la geometría es una ciencia natural sólo en cuento a su origen. El empirismo de Hilbert consiste en sostener que los hechos, leyes y conceptos básicos que están en la base de la geometría no pueden ser adquiridos a través del "pensamiento puro", sino que para ello es necesario el material proporcionado por la experiencia y la intuición. En cambio, no radicaliza su posición empirista exigiendo que todos los conceptos primitivos y axiomas de su teoría geométrica (axiomatizada) se correspondan con objetos y hechos empíricamente observables. Ésta es una diferencia importante respecto de otras posiciones radicalmente empiristas, como por ejemplo el programa de Pasch para la fundamentación de la geometría. ${ }^{12}$

Otra diferencia importante de esta imagen de la geometría, en relación a otras posiciones empiristas del siglo XIX, reside en que Hilbert no se muestra mayormente preocupado por el problema de explicar cómo es posible determinar o decidir empíricamente cuál de las diferentes teorías geométricas consistentes constituye la descripción "correcta" del espacio físico; en efecto, éste era el problema central que guiaba la forma de empirismo geométrico defendida por von Helmholtz. ${ }^{13}$ Por el contrario, el énfasis de Hilbert está puesto en la naturaleza "sintética" de los conceptos geométricos, y en la necesidad de realizar un examen axiomático de nuestras intuiciones geométricas.

Finalmente, a pesar de adoptar una posición empirista que concibe a la geometría cuanto a su origen como una ciencia natural, Hilbert pretende permanecer neutral en lo que se refiere al estatus epistemológico de esta intuición geométrica, advirtiendo que no se pronunciará respeto de esta difícil cuestión:

Finalmente podemos designar a nuestra tarea como un análisis lógico de nuestra intuición; la pregunta acerca de si nuestra intuición espacial tiene un origen a priori o empírico, permanecerá aquí sin discutir. (Hilbert 1898/1899b, p. 303)14

\section{El nuevo método axiomático formal}

Con esta imagen de la geometría de fondo, Hilbert emprende por primera vez la tarea de axiomatizar la geometría euclídea en un curso titulado "Los fundamentos

\footnotetext{
12 Esta posición empirista radical se manifiesta, por ejemplo, en el hecho de que Pasch toma a los segmentos lineales (finitos) como concepto primitivo de su sistema axiomático para la geometría proyectiva, dado que las líneas - que pueden "imaginadas como infinitamente extendidas - no se corresponden con ningún objeto observable (Cf. Pasch 1882, p. 4). Asimismo, Pasch rechaza la inclusión del axioma de Arquímedes en su sistema axiomático, en tanto considera que es un principio que no está justificado empíricamente. Sobre el "programa de Pasch" puede verse Schlimm (2010).

13 Sobre el empirismo de von Helmholtz y otras concepciones empirista de la geometría en el siglo XIX pueden consultarse Torretti (1984) y Gray (2007).

14 Una declaración similar se encuentra en Hilbert (1891), p. 27 y Hilbert (1902), p. 521.
} 
de la geometría" (Hilbert 1893/1894), correspondiente al semestre de invierno de 1893/1894. En este trabajo el método axiomático no está todavía tan desarrollado como en sus cursos posteriores. En particular, las investigaciones metageométricas, la novedad más importante introducida en Fundamentos de la geometría, no están aquí presentes. Sin embargo, en este primer abordaje axiomático a la geometría las ideas centrales del método axiomático abstracto o formal aparecen explícitamente formuladas.

En primer lugar, Hilbert señala que la descripción y organización de la totalidad de los hechos geométricos es llevada a cabo por medio de la construcción de un "entramado de conceptos" [Fachwerk von Begriffen], al que se le impone la condición de incluir o representar la totalidad de los hechos geométricos que componen la geometría euclídea elemental. ${ }^{15}$ Sin embargo, Hilbert entiende ahora que este entramado de conceptos no constituye una descripción directa o inmediata del espacio físico, sino que su sistema axiomático para la geometría puede tener múltiples realizaciones o "interpretaciones":

En general debe afirmarse: nuestra teoría proporciona sólo un esquema [Schema] de conceptos, conectados entre sí por las invariables leyes de la lógica. Se deja librado al entendimiento humano [menschlicher Verstand] cómo aplicar este esquema a los fenómenos, cómo llenarlo de material [Stoff]. Ello puede ocurrir de diversas maneras: pero siempre que los axiomas sean satisfechos, entonces los teoremas son válidos. Cuanto más fácil y más variadas son las aplicaciones, tanto mejor es la teoría.

Cada sistema de unidades y axiomas que describe completamente los fenómenos está tan justificado como cualquier otro. Mostrar sin embargo que el sistema axiomático aquí especificado es, respecto de cierto punto de vista, el más simple posible. (Hilbert 1893/1894, p. 104)

Hilbert formula de este modo, ya en 1894, la idea central de su nueva concepción abstracta método axiomático. Una axiomatización formal de la geometría arroja un "esquema de conceptos", o mejor, un entramado de relaciones lógicas entre conceptos, que se halla separado de la realidad, en el sentido de que la teoría axiomática no intenta ofrecer una descripción directa del espacio físico. Los 'puntos', 'líneas' y 'planos' de su teoría geométrica no deben ser entonces identificados como los objetos geométricos habituales, accesibles a la intuición geométrica. Por el contario, ellos constituyen más bien un conjunto de objetos "pensados", cuyas propiedades (matemáticas) fundamentales son establecidas de un modo riguroso y completo en los axiomas de la geometría.

De acuerdo con esta nueva concepción axiomática abstracta, los axiomas de la geometría no conforman un conjunto de verdades evidentes acerca de los objetos

15 Cf. Hilbert (1893/1894), p. 72. 
geométricos intuitivos, aun cuando su origen se encuentre en la experiencia y la intuición. Las teorías geométricas no pueden ser entonces verdaderas o falsas por representar correctamente, o por fallar en representar, cierto conjunto de objetos (físicos) o dominio determinado. Por el contrario, la consistencia es ahora la condición fundamental que debe garantizarse de toda teoría axiomática. La realidad no determina a la teoría geométrica, en el sentido de que la limita a lo que, a primera vista, está intuitiva y empíricamente justificado; en cambio, el requisito fundamental establecido consiste en exigir que el sistema de axiomas no pueda conducir a contradicciones. En Fundamentos de la geometría Hilbert demuestra esta última condición exhibiendo un "modelo" aritmético para sus axiomas geométricos, construido sobre un sub-cuerpo pitagórico (minimal) de los números reales. ${ }^{16}$

Una consecuencia inmediata de esta nueva concepción axiomática abstracta consiste en que la pretensión de que la geometría pueda ofrecer una descripción directa de la forma o el comportamiento de los cuerpos en el espacio debe ser rechazada. Sin embargo, Hilbert reproduce en sus cursos esta definición de la geometría, articulando su posición de la siguiente manera: en primer lugar, afirma que su estudio axiomático abstracto nos proporciona un conocimiento preciso de la estructura - i.e., de las propiedades lógicas de los axiomas y su relación con los teoremas fundamentales - de la geometría euclídea. En este sentido, el sistema axiomático obtenido por medio de la axiomatización arroja un entramado de relaciones lógicas entre conceptos que no posee una relación directa o inmediata con un dominio fáctico intuitivo. Mas, en lo que respecta al lugar de la geometría dentro de las disciplinas matemáticas fundamentales, ésta sigue siendo una teoría que en sus orígenes está esencialmente ligada a la experiencia y a nuestra intuición espacial.

Esta concepción de la geometría como la ciencia natural más completa resulta fundamental para comprender la función que Hilbert le confiere, en esta etapa temprana, a su nuevo método axiomático, a saber: a través del tratamiento axiomático formal la geometría se convierte, con su contenido empírico factual, en una teoría matemática pura:

También la geometría surge [como la mecánica] de la observación de la naturaleza, de la experiencia, y en ese sentido es una ciencia experimental. En mi curso sobre geometría euclídea me introduciré en este tema más de cerca. Pero sus fundamentos experimentales son tan irrefutables y tan generalmente reconocidos, han sido confirmados en un grado tal, que no se requiere de ninguna prueba ulterior. Todo lo que se necesita es derivar estos fundamentos de un conjunto mínimo de axiomas independientes y así construir todo el edificio de la geometría por medios puramente lógicos. De este modo [i.e. por medio del tratamiento axiomático], la geometría se vuelve una ciencia matemática pura. (Hilbert 1898, pp. 1-2)

16 Cf. Hilbert (1899), §9. 
El grado de avance alcanzado por la geometría vuelve además imprescindible el análisis axiomático, en el modo en que ahora es reformulado. En un pasaje que anticipa las ideas presentadas casi veinte años más tarde en su conferencia "El pensamiento axiomático" (Hilbert 1918), Hilbert subraya esta necesidad de la siguiente manera:

Cuanto más se acerca una ciencia natural a su objetivo: "la deducción lógica de todos los hechos que pertenecen a su campo a partir de ciertas proposiciones fundamentales", tanto más necesario se vuelve investigar estos mismos axiomas con precisión, indagar sus relaciones mutuas, reducir su número tanto como sea posible, etc. (Hilbert 1898/1899b, p. 302)

En suma, la temprana concepción de la geometría de Hilbert puede ser caracterizada a través de dos componentes fundamentales, a saber, $i$ ) una posición axiomática formal completamente articulada, que concibe el resultado de una axiomatización formal como un entramado de conceptos en donde los términos y relaciones básicas no poseen una referencia (intuitiva) fija, sino que pueden recibir diversas interpretaciones, tanto dentro de otras teorías matemáticas o físicas, como así también interpretaciones empíricas; ii) una posición empirista respecto del origen de la geometría y de su lugar dentro de las distintas teorías matemáticas.

Ahora bien, estas notas de clases no sólo nos permiten distinguir estos dos componentes fundamentales de la concepción hilbertiana de la geometría, sino que además encontramos allí observaciones y reflexiones muy interesantes respecto de cómo estos dos aspectos se relacionan. Hilbert ofrece en sus cursos una clara explicación de cómo debe concebirse la relación entre el conjunto de hechos [Tatsachen] geométricos fundados en la experiencia y la intuición, que conforma el acervo fundamental sobre el que se erige nuestro conocimiento geométrico, y la estructura relacional o esquema de conceptos, que es el producto de la axiomatización formal. Esta explicación constituye, como veremos, un elemento muy importante de su temprana concepción del método axiomático formal. Más aún, el modo en que Hilbert concibe la conexión entre su sistema axiomático formal para la geometría euclídea y el conjunto de hechos geométricos no sólo resulta sumamente interesante, sino que también revela una actitud en cierta medida todavía un poco tradicional respecto de la naturaleza del conocimiento geométrico.

\section{Geometría, formalismo y método axiomático}

Como es bien sabido, la presentación de la geometría como un sistema axiomático formal llevada a cabo por Hilbert, sumada a su posterior programa 'finitista' para la fundamentación de la aritmética y el análisis, han contribuido a formar una 
imagen excesivamente formalista de su concepción de la geometría, que todavía sigue siendo reproducida en las exposiciones de carácter general. De acuerdo con esta imagen, que podemos llamar formalista radical o extrema, el objetivo central del método axiomático de Hilbert es defender una concepción de toda la matemática clásica como una colección de sistemas deductivos abstractos completamente formalizados, construidos a partir de un conjunto de axiomas arbitrariamente escogidos y sin un significado intrínseco. Más aún, para los defensores de esta interpretación, la idea detrás del método axiomático hilbertiano es que la matemática consiste básicamente en el estudio de los formalismos, entendidos como el esquema de signos o símbolos sin significado, sujeto a un conjunto de reglas estipuladas, que componen el sistema axiomático. ${ }^{17} \mathrm{Sin}$ embargo, la imagen de la geometría que presenta Hilbert en sus cursos poco tiene que ver con estas posiciones excesivamente formalistas; sostendré a continuación que la temprana concepción axiomática de la geometría se opone claramente a este tipo de lecturas radicalmente formalistas.

Por otro lado, el término "formalismo" ha sido utilizado de un modo diferente para caracterizar la posición de Hilbert, en esta etapa geométrica. El formalismo hilbertiano en este segundo sentido, que podríamos llamar moderado ${ }^{18}$, afirma que toda teoría matemática axiomatizada consiste en un entramado o esquema de relaciones lógicas entre conceptos, que no está ligado a un determinado dominio fijo, sino que diversos dominios de objetos pueden tener en común. Un sistema de axiomas no constituye así un conjunto de proposiciones verdaderas acerca de un dominio particular de objetos, sino que las proposiciones o teoremas de una teoría axiomática deben ser entendidos en un sentido hipotético, esto es, como siendo verdaderas para cualquier "interpretación" de los términos y relaciones básicas en las que los axiomas son satisfechos. ${ }^{19}$

Luego, ya en su primer curso de 1894, Hilbert formula de este modo la tesis fundamental de su nueva concepción abstracta del método axiomático. Esta última manera de caracterizar la empresa hilbertiana describe entonces correctamente el

\footnotetext{
17 Frege fue uno de los primeros en impulsar este tipo de lectura, no sólo en su célebre controversia epistolar con Hilbert, sino sobre todo en una serie de artículos que esta última originó (Cf. Frege 1903, 1906). Asimismo, declaraciones similares fueron repetidas por Weyl en diversos trabajos, por ejemplo, en Weyl (1925). Finalmente, la interpretación formalista radical se encuentra paradigmáticamente representada en el clásico y difundido artículo de Dieudonné (1971), vocero del mítico grupo de matemáticos franceses Bourbaki.

18 Lassalle Casanave (1996) propone distinguir entre interpretaciones formalistas extremas e interpretaciones formalistas moderadas del programa finitista de Hilbert para la fundamentación de la aritmética y el análisis. Esta distinción, sin embargo, no coincide exactamente con las interpretaciones formalista se Hilbert en la llamada "etapa geométrica."

19 Bernays (1922a, 1922b) fue el primero en proponer esta interpretación, en una serie de artículos que pretendían exponer las recientes contribuciones de Hilbert a los fundamentos de la matemática. En una época más contemporánea esta lectura ha sido presentada por Detlefsen (1993), Mancosu (1998), y en la literatura castellana por Torres Alcaraz (2009).
} 
"giro metodológico" que éste le imprime a la idea de axiomática, en este período inicial. Sin embargo, en lo que sigue intentaré llamar la atención sobre otros aspectos de su abordaje axiomático a la geometría, que considero relevantes para alcanzar una imagen más equilibrada y mejor contextualizada de su concepción del método axiomático formal en esta etapa temprana. Puntualmente, estos elementos están vinculados tanto a su concepción de la geometría, como a su modo de entender el proceso de realizar una axiomatización (formal) de una teoría matemática.

\section{1 "Una imagen de la realidad geométrica"}

En sus cursos sobre geometría Hilbert revela un aspecto muy interesante de su concepción del método axiomático, que en cierta medida resulta llamativo si se lo considera desde una perspectiva estrictamente axiomática formal. Este aspecto consiste en una especie de condición o criterio (informal) de adecuación, según el cual sus sistemas axiomáticos abstractos, y en particular el sistema para la geometría euclídea, deben conservar una conexión o vinculación con los hechos intuitivos básicos que están la base de esta teoría matemática, esto es, con los hechos geométricos fundamentales fundados en la experiencia y la intuición. Si bien el resultado de su axiomatización de la geometría es un entramado de relaciones lógicas entre conceptos, que diversos dominios de objetos pueden tener común, Hilbert enfatiza al mismo tiempo que en su construcción del sistema axiomático buscó explícitamente mantener o reflejar de algún modo un paralelismo con los hechos intuitivos básicos de la geometría. Un primer indicio de esta condición o criterio (informal) de adecuación se encuentra en la descripción, presentada por Hilbert en sus manuscritos, de la empresa de realizar una axiomatización de la geometría como la tarea de ofrecer "una imagen $[$ Bild $]$ de la realidad geométrica":

Empleando una expresión de Hertz (en la introducción a los "Principios de la mecánica"), podemos formular nuestra pregunta principal como sigue: ¿cuáles son las condiciones necesarias, suficientes e independientes entre sí, que deben establecerse respecto de un sistema de cosas [Dinge], para que a cada una de estas cosas le corresponda un hecho geométrico, e inversamente, para que por medio del mencionado sistema de cosas sea posible una descripción completa u organización de todos los hechos geométricos; o para que nuestro sistema se convierta en una imagen de la realidad geométrica? (Hilbert 1898/1899b, p. 303. El énfasis es mío) ${ }^{20}$

Hilbert compara aquí su empresa de axiomatizar la geometría con la presentación de la mecánica realizada por Heinrich Hertz (1857-1894), en su notable obra

20 Referencias similares a la teoría pictórica de Hertz se encuentran en Hilbert (1893/1894), pp. 72-73; (1902), p. 51. 
Principios de la mecánica (1894). ${ }^{21}$ En particular, sugiere que la "teoría pictórica" o Bildtheorie de Hertz resulta significativa para comprender el espíritu con el cual emprende la tarea de axiomatizar la geometría. Esta comparación es posible ya que para el matemático alemán la geometría se encuentra, en lo que refiere a sus bases epistemológicas, más cerca de teorías físicas como la mecánica, que de teorías matemáticas puras como la aritmética y el análisis. Con la utilización del término "imagen" [Bild], Hilbert advierte que tanto sus axiomas ${ }^{22}$, como el sistema axiomático en su conjunto, deben ser entendidos como representaciones intelectuales o puramente conceptuales de un conjunto de fenómenos, i.e., el conjunto de los hechos geométricos. Los elementos de esta imagen son tres sistemas de objetos cualesquiera, que Hilbert designa también como "objetos del pensamiento" [Gedankendinge], para aclarar que los "puntos", "líneas" y "planos" pertenecen a un nivel exclusivamente conceptual, y por lo tanto deben ser diferenciados de los "puntos", "líneas" y "planos" reales o de la intuición. La alusión de Hilbert a la Bildtheorie sugiere que su nueva concepción del método axiomático coincide esencialmente con la posición de Hertz en lo que respecta al cambio del estatus epistemológico de las teorías científicas, a saber: éstas deben dejar de ser consideradas como una descripción directa e inmediata de la naturaleza, para comenzar a ser vistas como construcciones intelectuales, como "imágenes" [Bilder] que nos formamos de los fenómenos.

Ahora bien, una cuestión central de esta referencia es que el sistema axiomático que se pretende construir debe ser una imagen de la realidad geométrica. Hilbert nunca aclara de un modo preciso que es lo que entiende por esta expresión. Sin embargo, podemos presumir que con ella no se alude primariamente a los hechos empíricos que están en la base de la geometría, sino más bien al conjunto de conocimientos o "verdades geométricas" que se han llegado a reconocer y aceptar generalmente por medio de la acumulación de demostraciones. ${ }^{23}$ Considerando que lo que se intenta reconstruir axiomáticamente es la geometría euclídea elemental, podría decirse entonces que la "realidad geométrica" es el acervo de conocimientos, con una fuerte base intuitiva, conseguidos por esta disciplina en su etapa acrítica o intuitiva. ${ }^{24}$ De este modo, al caracterizar su empresa axiomática con la tarea de proporcionar una "imagen de la realidad geométrica", Hilbert destaca la importancia desde un inicio de las fuentes fundamentales de conocimiento geométrico en

\footnotetext{
21 Sobre la influencia de Hertz en la temprana concepción axiomática de Hilbert puede verse Giovannini (2012).

22 Más temprano en este mismo curso Hilbert señala: "los axiomas [de la geometría] son, como diría Hertz, imágenes o símbolos en nuestra mente" (Hilbert 1893/1894, p. 74).

23 Hilbert habla de "verdades geométricas", por ejemplo, en Hilbert (1898/1899a), p. 236.

24 En 1893 Hilbert distingue tres períodos de desarrollo, clara y fácilmente reconocibles, en la historia de cualquier teoría matemática: el naive o intuitivo, el formal y el crítico. El método axiomático, comprensiblemente, se identifica con el período crítico (Cf. Hilbert 1896, p. 383).
} 
el proceso de axiomatizar la geometría. No se trata así de jugar con un conjunto cualquiera de postulados o axiomas, para ver qué proposiciones o teoremas es posible obtener de allí exclusivamente por medio de deducciones lógicas. Antes bien, lo que se busca es alcanzar una representación lógicamente más perspicua y consistente, de una disciplina para Hilbert enraizada en sus orígenes en la experiencia y la intuición.

\subsection{Axiomatización e intuición}

Hilbert resalta aun de un modo más explícito la importancia de que su sistema axiomático formal para la geometría elemental conserve una conexión con los hechos geométricos básicos, fundados en la experiencia y la intuición, en las notas de clases para el curso Principios lógicos del pensamiento matemático (Hilbert 1905a, 1905b). Este curso dictado en el semestre de verano de 1905 constituye la exposición más acabada del método axiomático formal, correspondiente a este período temprano. La sección dedicada al sistema axiomático para la geometría euclídea elemental se inicia con la siguiente caracterización:

El objetivo de toda ciencia es, en primer lugar, establecer un esquema de conceptos basado en axiomas a cuya misma concepción somos naturalmente guiados por la intuición y la experiencia. Idealmente, todos los fenómenos de un dominio dado aparecerán como una parte del esquema y todos los teoremas que pueden ser derivados de los axiomas encontrarán su expresión allí.

Así, si queremos establecer un sistema de axiomas para la geometría, el punto de partida debe sernos dado por los hechos intuitivos de la geometría y éstos deben corresponderse con el esquema que debe ser construido. Los conceptos obtenidos de este modo, sin embargo, deben ser considerados como separados completamente de la experiencia y la intuición. (Hilbert 1905b, pp. 36-37)

Hilbert indica que sus sistemas axiomáticos son formales, y en cuanto tales deben ser considerados como "separados de la intuición", dado que los términos y relaciones básicas no están ligados a una interpretación intuitiva fijada de antemano. Sin embargo, al mismo tiempo aclara que ello no significa que la intuición no desempaña más un papel en la teoría geométrica axiomática. Por el contrario, la intuición y la experiencia resultan esenciales para la selección de los principios básicos sobre los cuales se construirá deductivamente todo nuestro conocimiento geométrico. Asimismo, la función de la intuición no se circunscribe únicamente a sugerir la elección de los axiomas de la teoría, sino que además resulta fundamental para que el sistema axiomático pueda ser aplicado a la realidad. Hilbert señala a continuación que en la construcción de los sistemas axiomáticos (formales) debe 
buscarse que el esquema conceptual resultante conforme una analogía con nuestras intuiciones más básicas y con los hechos de la experiencia. Este requisito es formulado de la siguiente manera:

En la presentación axiomática de la aritmética nos hemos alejado totalmente del concepto original de número y con ello nos hemos separado de toda intuición. Los números se convirtieron para nosotros solamente en un entramado de conceptos, a los que por supuesto sólo somos guiados por la intuición; sin embargo, podemos operar con este entramado sin recurrir a la ayuda de la intuición. Ahora bien, para que este sistema conceptual pueda ser aplicado a las cosas que nos rodean, es necesario que sea construido de tal manera que forme una completa analogía con nuestras intuiciones más simples y con los hechos de la experiencia. (Hilbert 1905b, p. 27. El énfasis es mío)

Estas afirmaciones resultan sumamente importantes para comprender cómo concebía Hilbert, en esta etapa temprana, la naturaleza del método axiomático formal, y especialmente, cuál era el significado y la finalidad fundamentales que encontraba en su abordaje axiomático formal a la geometría. En primer lugar, es claro que para Hilbert su análisis axiomático de la geometría de ningún modo consistía en el estudio de las consecuencias lógicas que podían ser derivadas de un conjunto de postulados dados, en principio elegidos con completa libertad y sin un significado intrínseco. 25 Por el contrario, una razón fundamental para realizar un análisis axiomático formal era profundizar nuestro conocimiento, y perfeccionar la claridad lógica, de una teoría matemática en un estado avanzado de su desarrollo.

En segundo lugar, en esta etapa inicial Hilbert tampoco pensaba que la tarea de llevar a cabo una axiomatización formal se limitaba exclusivamente a reducir un dominio de conocimiento determinado a un esquema de relaciones lógicas entre conceptos, cuya validez debía ser luego justificada por medio del estudio de las propiedades metalógicas del sistema de axiomas (consistencia, independencia, completitud). ${ }^{26}$ Antes bien, junto con aquellos conocidos criterios de adecuación establecidos para todos los sistemas axiomáticos, Hilbert encontraba además importante que el sistema de axiomas propuesto no pierda completamente su conexión con estas fuentes originales del conocimiento geométrico. En gran parte, este requisito se explica en virtud de que en este período inicial Hilbert consideraba realmente a la geometría como una teoría matemática fundada en gran medida en la intuición y en la experiencia.

\footnotetext{
25 Ésta fue, por ejemplo, la enseñanza que Poincaré pareció extraer del trabajo de Hilbert. Véase en particular su reseña a Fundamentos de la geometría (Poincaré 1902). El propio Hilbert se encargó de rechazar, en un período bastante posterior, este tipo de lecturas excesivamente formalista. La declaración quizás más explícita se encuentra en el curso Natur und mathematisches Erkenen (Hilbert 1992), dictado en 1919.

26 Las investigaciones metageométricas de Hilbert fueron, sin embargo, una de su contribuciones más originales y matemáticamente fructíferas a la axiomática moderna.
} 
En este preciso sentido, aunque el proceso mismo de axiomatización formal consistía en una proyección desde un plano intuitivo inicial a un nivel puramente conceptual, Hilbert juzgaba como esencial que su sistema de axiomas conserve de algún modo un cierto paralelismo con el contenido intuitivo-empírico de esta teoría. La supuesta arbitrariedad con la que en principio podían ser elegidos los axiomas y términos primitivos de un sistema axiomático, estaba limitada de hecho por la exigencia de que éstos permanezcan lo más cerca posible de los hechos básicos de nuestra intuición geométrica. De esta manera, esta idea se relaciona con una de las afirmaciones más controvertidas de su libro Fundamentos de la geometría, a saber: el análisis axiomático formal de la geometría consiste al mismo tiempo en un análisis lógico de la intuición.

\section{5. "Un análisis lógico de la intuición"}

En la introducción de Fundamentos de la geometría (1899), Hilbert alude al problema que será su objeto de indagación de la siguiente manera:

La geometría necesita para su construcción lógica - del mismo modo que la aritmética - sólo unos pocos y simples hechos fundamentales. A estos hechos fundamentales se los denomina axiomas. El establecimiento de los axiomas de la geometría y la investigación de sus conexiones es una tarea que, desde Euclides, ha sido discutida en numerosos excelentes tratados de la literatura matemática. Esta tarea consiste en el análisis lógico de nuestra intuición espacial. (Hilbert 1899, p. 3. El énfasis es mío.)

Esta afirmación ha sido considerada como inadecuada y confusa en el contexto de su libro de carácter puramente matemático, fundamentalmente en virtud de que los axiomas allí propuestos para la geometría euclídea conforman un sistema formal. Sin embargo, Hilbert la repite constantemente en sus cursos, indicando que se trata de un elemento importante de su concepción axiomática de la geometría. 27 Una referencia interesante al respecto se encuentra en el curso de 1898/1899, en donde explica las diferencias entre su abordaje axiomático a la geometría y los abordajes sintéticos y analíticos o algebraicos de la siguiente manera:

A partir de lo dicho se esclarece la relación de este curso con aquellos sobre geometría analítica y geometría proyectiva (sintética). En ambas disciplinas las preguntas fundamentales no son tratadas. En la geometría analítica se comienza con la introducción del número; por el contrario nosotros habremos de investigar con precisión la justificación para ello, de modo que en nuestro caso la introducción del número se producirá al final. En la geometría proyectiva se apela desde el principio a la intuición, mientras que noso-

27 Véase Hilbert (1898/1899b), p. 303; Hilbert (1902), p. 541. 
tros queremos analizar la intuición, para reconstruirla, por decirlo de algún modo, en sus componentes particulares [einzelne Bestandteile]. (Hilbert 1898/1899b, p. 303. El énfasis es mío)

En un sentido estricto, las investigaciones de Hilbert son un análisis lógico de los axiomas, no de la intuición. Ellas nos proporcionan una descripción matemáticamente exacta y completa de la estructura lógica de esta teoría matemática, i.e., de cuáles son las condiciones o principios necesarios y suficientes que deben ser postulados para construir esta teoría y de las relaciones lógicas de los axiomas entre sí y también con los teoremas fundamentales. Mas, en este período inicial, Hilbert considera todavía que su examen axiomático de la geometría euclídea es, al mismo tiempo, un análisis de las fuentes originales del conocimiento geométrico, i.e., de la experiencia y de nuestra intuición geométrica. Estas investigaciones contribuyen por lo tanto a esclarecer qué principios y proposiciones son responsables de varias de las partes centrales de nuestro conocimiento geométrico intuitivo.

Hilbert concibe así su análisis axiomático formal de la geometría como un suerte de reconstrucción racional o conceptual, que supone un abandono de toda interpretación intuitiva fija y de la intuición geométrica en pos del procedimiento de construcción de "modelos" (aritméticos), pero que a su vez conserva un vínculo con los hechos básicos de la intuición, en tanto nos permite distinguir allí "qué elementos pertenecen a la experiencia y qué hechos son [sus] consecuencias lógicas" (Hilbert 1898/1899a, p. 222).

Ahora bien, que el examen axiomático constituya un análisis lógico de la intuición, no significa para Hilbert que el sistema axiomático propuesto debe ser considerado como una descripción directa y exacta de un determinado dominio intuitivo dado:

En cierto modo hemos dado en este curso una teoría de la geometría; deseamos ahora hacer una observación acerca de la aplicación de esta teoría a la realidad. Las proposiciones geométricas nunca son válidas en la naturaleza con completa exactitud, porque los axiomas nunca son satisfechos [erfüllt] por los objetos. Esta carencia en la correspondencia reside en la esencia de toda teoría, pues una teoría, que se corresponda hasta en detalle con la realidad, sería sólo una descripción exacta del objeto. (Hilbert $1898 / 1899$ b, p. 391) 28

Hilbert reconoce que las distintas interpretaciones empíricas que pueden proponerse del sistema axiomático formal para la geometría sólo pueden tener un carácter aproximativo. Ello significa que el "esquema o entramado de conceptos", que es el resultado de la axiomatización, no puede estar de ningún modo limitado por lo que a primera vista parece estar empírica o intuitivamente justificado. En tanto que

28 Véase también Hilbert (1898/1899b), p. 401 y Hilbert (1898/1899a), p. 283. 
la teoría geométrica formal no se refiere de un modo directo a la realidad [Wirklichkeit], no puede decirse entonces que una interpretación particular del sistema axiomático debe ser privilegiada por sobre otras. Por el contrario, la geometría puede aprender de la intuición, la observación y de la investigación empírica, pero no debe ser su esclava, incluso cuando la intuición juegue un rol decisivo en el establecimiento del conjunto de hechos que constituyen el dominio básico de la geometría. Dicho de otro modo, aunque en las investigaciones geométricas nos vemos guiados constantemente por la intuición geométrica y nos planteamos preguntas y problemas sugeridos por la intuición, al final es el análisis axiomático formal el que instruye a la intuición, no al revés. ${ }^{29} \mathrm{Y}$ en definitiva, el hecho de que la intuición geométrica requiera de un análisis axiomático formal, se explica en razón de que Hilbert no la considera una fuente segura o totalmente fiable de conocimiento geométrico. Esta última afirmación nos lleva a intentar precisar qué es lo que entiende Hilbert por "intuición geométrica", noción invocada con insistencia a lo largo de sus cursos.

\section{La noción de "intuición geométrica"}

La cuestión de la naturaleza, el contenido y el estatus epistemológico de nuestra "intuición geométrica" es un problema sobre el que Hilbert no profundiza en ningún momento en sus cursos sobre geometría, en gran parte debido a que él mismo advierte que se trata de un problema estrictamente filosófico que excede sus investigaciones de carácter puramente matemático.30 Sin embargo, aunque no encontramos en las fuentes que venimos analizando una elucidación filosófica mínima de esta noción central, considero que es posible realizar algunas observaciones al respecto.

En primer lugar, en virtud de su concepción de la geometría como una ciencia natural, resulta lícito inferir que Hilbert entiende la naturaleza de nuestra intuición espacial en términos más bien empiristas; en efecto, si la intuición espacial que está detrás de nuestro conocimiento geométrico fuera pura o a priori, entonces la geometría podría ser considerada una teoría matemática pura en lo que respecta a sus bases o fuentes epistemológicas. En diversas ocasiones, especialmente al referirse al origen de los axiomas de la geometría, Hilbert parece concebir de este manera la intuición espacial, equiparándola con la mera observación o percepción de simples

\footnotetext{
${ }^{29}$ A propósito de esta conclusión, véanse los ejemplos de las investigaciones axiomáticas de Hilbert analizados por Hallett (2008).

30 Como hemos visto, Hilbert señala en diversas oportunidades a lo largo de sus cursos que "la pregunta, acerca de si nuestra intuición espacial tiene un origen a priori o empírico, permanecerá aquí sin discutir" Hilbert (1898/1899b), p. 303.
} 
configuraciones de objetos en el espacio: "En efecto la geometría de los antiguos surge también de la intuición de las cosas [Anschauung der Dinge] en el espacio, tal como se ofrece en la vida cotidiana [tägliches Leben]" (Hilbert 1891, p. 23). O de la misma manera: "El axioma corresponde a una observación, como puede verse fácilmente en las esferas, reglas y superficies de cartulina [Pappdeckeln]" (Hilbert 1894, p. 74). Asimismo, en cuanto a la forma o estructura de esta intuición espacial, es claro que en esta etapa temprana previa al surgimiento de la teoría general de la relatividad, Hilbert parece no tener dudas respecto de que la forma en la que percibimos las relaciones espaciales se corresponde exactamente con la geometría euclídea. ${ }^{31}$

Ahora bien, es dable notar que en diversos pasajes de sus cursos citados anteriormente, Hilbert distingue la experiencia y la intuición geométrica como dos fuentes distintas, complementarias pero independientes, de nuestro conocimiento geométrico. ${ }^{32}$ Ello permitiría pensar que concibe la intuición geométrica al mismo tiempo en un sentido más bien diferente al anterior.

En mi opinión, Hilbert también se refiere a la "intuición geométrica" en un sentido un poco más sutil, ligado más bien a la práctica matemática. La intuición geométrica en este sentido puede ser concebida como una cierta habilidad o capacidad, que puede ser instruida y desarrollada, para percibir o captar inmediatamente relaciones geométricas fundamentales exhibidas generalmente en las construcciones geométricas y diagramáticas. Este modo de entender la intuición geométrica tiene lugar en el contexto del estudio y la práctica de la geometría en un nivel "informal" o "intuitivo", cuando los objetos geométricos primitivos ('puntos', 'líneas', 'planos', etc.) y las relaciones básicas son concebidas efectivamente a partir de su significado geométrico intuitivo habitual. La "intuición geométrica" en este segundo sentido está así ligada al razonamiento geométrico fuertemente basado en diagramas que es propio de lo que Hilbert y Bernays (1934) llaman más tarde "axiomática material" [inhaltliche Axiomatik], en particular al tipo de razonamiento diagramático que sigue la tradición iniciada por los Elementos de Euclides. Más aún, una característica esencial de la "intuición geométrica", de acuerdo con este segundo sentido, consiste en la capacidad de visualizar diversas configuraciones de objetos y situaciones geométricas fundamentalmente a partir de la utilización de figuras y

\footnotetext{
31 Véase especialmente Hilbert (1893/1894), p. 119-120 y Hilbert (1905b), p. 67. Corry $(2004,2006)$ ha analizado cómo la teoría general de la relatividad, en particular las novedosas relaciones entre la gravitación y la geometría establecidas por esta teoría, afectaron la imagen de la geometría defendida por Hilbert.

32 Corry (2006) sostiene que, aunque el propio Hilbert aclara que no se pronunciará sobre esta cuestión, esta diferenciación entre experiencia e intuición permitiría pensar que ésta última reviste un carácter a priori, en un sentido kantiano. Por otro lado, en Torres Alcaraz (2009) y Majer (2006) se encuentran dos exámenes de la postura de Hilbert en esta primera etapa geométrica, en comparación con la filosofía de la geometría de Kant.
} 
diagramas, por medio de los cuales es posible una comprensión más inmediata del contenido 33 de las proposiciones (axiomas, teoremas, etc.) geométricas.

Desde mi punto de vista, Hilbert tiene en mente esta noción más refinada cuando subraya una y otra vez la importancia capital de la intuición geométrica para una axiomatización (formal) exitosa de la geometría elemental. Un claro ejemplo es el siguiente pasaje correspondiente a las notas de clases para su curso "Principios lógicos del pensamiento matemático" de 1905:

Cuando uno se pregunta por el lugar, dentro de todo el sistema, de un teorema conocido desde antaño como el de la igualdad de los ángulos de la base de un triángulo, entonces naturalmente se debe apartar de las creencias tradicionales y de la intuición, y aplicar solamente las consecuencias lógicas de los axiomas presupuestos. Para asegurarse de ello, a menudo se ha hecho la sugerencia de evitar los nombres usuales de las cosas, ya que éstos pueden desviarnos, a través de las numerosas asociaciones con los hechos de la intuición, de la rigurosidad lógica. Se ha sugerido así introducir en el sistema axiomático nuevos nombres para 'puntos', 'líneas', 'planos', etc.; nombres que recuerden solamente lo que ha sido establecido en los axiomas. Se ha propuesto incluso que palabras como 'igual', 'mayor', 'menor', sean reemplazadas por formaciones arbitrarias de palabras, como 'a-rig', 'b-rig', 'a-rung', 'be-rung'. Ello es de hecho un buen medio pedagógico para mostrar que un sistema axiomático sólo se ocupa de las propiedades establecidas en los axiomas y de nada más. Sin embargo, en la práctica este procedimiento no es ventajoso, e incluso no está realmente justificado. En efecto, uno siempre debe guiarse por la intuición al formular un sistema axiomático y uno siempre tiene a la intuición como una meta [Zielpunkt]. Por lo tanto, no es defecto alguno si los nombres nos recuerdan siempre, e incluso hacen más fácil recordar, el contenido de los axiomas, puesto que se puede evitar fácilmente la intromisión de la intuición en las investigaciones lógicas, al menos con un poco de cuidado y práctica. (Hilbert 1905a, 87-88. El énfasis es mío.)

La intuición que sirve como guía y que debe ser tenida como una meta [Zielpunkt] en el proceso de la construcción de un sistema axiomático formal para la geometría (elemental) es así la intuición geométrica que es ejercitada en el razonamiento geométrico basado en diagramas ${ }^{34}$ que tiene lugar originalmente en el

\footnotetext{
33 Analizando las discusiones en torno a la exigencia de la "pureza del método" en las investigaciones axiomáticas de Hilbert en el campo de la geometría, Arana y Mancosu (2012) distinguen entre una noción "informal" o "intuitiva" y otra "formal" del contenido de una proposición geométrica (axioma, teorema, etc.). El contenido de una proposición geométrica que se vuelve inmediatamente accesible a través de la intuición geométrica se corresponde así con la primera noción "informal" o "intuitiva", con lo que se alude a lo que "alguien con un entendimiento casual de la geometría sería capaz de comprender" (Arana y Mancosu 2012, p. 327). En cambio, la noción formal del contenido de una afirmación se identifica con "el rol inferencial de una proposición dentro de un sistema axiomático" (Íbid.). 34 Sobre la posición de Hilbert respecto del uso de diagramas en matemática, particularmente en relación al razonamiento basado en diagramas que propone Minkowski en su obra Geometrie der Zahlen (1896), puede verse Smadja (2012).
} 
contexto de la axiomática material, en donde los objetos y relaciones básicas de la geometría están ligados a su significado ‘intuitivo' habitual.35 Es oportuno señalar que esta relevancia epistemológica de la axiomática material para la axiomática formal, determinada a través del papel significativo que se le atribuye a la intuición geométrica, no es un elemento que se circunscribe a su temprana concepción axiomática de la geometría, sino que es un aspecto que Hilbert resalta explícitamente en una etapa posterior de sus trabajos sobre los fundamentos de la matemática:

La axiomática formal requiere necesariamente de la axiomática material como su complemento [Ergänzung], pues esta última proporciona en primer lugar la guía en la elección del formalismo; más aún, también [la axiomática material] aporta la indicación de cómo debe ser aplicada una teoría formal dada a un dominio de lo real [Gebiet der Tatsächlichkeit]". (Hilbert y Bernays 1934, p. 2)

Finalmente, el papel que Hilbert le asigna a la intuición geométrica en su temprana concepción axiomática de la geometría sin dudas contribuye a que su naturaleza y estatus epistemológico no sean debidamente especificados y mínimamente esclarecidos. En efecto, aunque la intuición es esencial para el establecimiento del conjunto de hechos geométricos fundamentales y sirve de guía en el proceso de axiomatización, es el análisis axiomático formal lo que en última instancia proporciona la justificación epistemológica de nuestro conocimiento geométrico. Por consiguiente, la concepción axiomática formal defendida por Hilbert resulta así compatible con las distintas maneras de concebir el contenido y el estatus epistemológico de nuestra intuición espacial. 36

\section{Consideraciones finales}

El objetivo de este trabajo ha sido reconstruir y analizar la temprana concepción axiomática de la geometría de Hilbert, utilizando principalmente sus notas manuscritas de clases para cursos universitarios sobre geometría, correspondientes al período 1891-1905. Estas fuentes nos han permitido reconocer una serie de consideraciones filosóficas y reflexiones metodológicas que subyacen a su axiomatización formal de la geometría euclídea, pero que sin embargo no resultan fácilmente reco-

\footnotetext{
35 Quizás esta misma noción de intuición geométrica es ilustrada posteriormente por Hilbert en su libro - en coautoría Cohn-Vossen - Anschauliche Geometrie (1932).

36 El estatus de la "intuición" es un problema que Hilbert tampoco resuelve definitivamente en su posterior programa 'finitista' para la fundamentación de la aritmética y el análisis (Cf. Mancosu 2010, cap. 2). Sin embargo, el papel que esta noción desempeña allí es diferente al que hemos identificado en la etapa geométrica, en tanto la intuición resulta ahora fundamental en la justificación epistemológica del conocimiento metamatemático.
} 
nocibles en el contexto de la exposición de carácter estrictamente matemático en Fundamentos de la geometría (1899).

En primer lugar, hemos mostrado que esta concepción experimenta una suerte de evolución desde el primer trabajo que Hilbert dedica a la geometría en 1891, hasta la discusión más detallada y completa sobre los fundamentos axiomáticos de la geometría que encontramos en este período inicial, correspondiente a un curso dictado en 1905. En sus primeros cursos Hilbert todavía caracteriza la geometría de un modo tradicional, al definirla como la ciencia que estudia las propiedades o forma de las cosas en el espacio. Los cursos posteriores exhiben, en cambio, una concepción axiomática abstracta de la geometría completamente desarrollada. Asimismo, nuestro análisis ha revelado que esta concepción formal del método axiomático estuvo acompañada por una posición empirista, según la cual los hechos básicos sobre los que se construye la geometría provienen de la experiencia y de una suerte de "intuición geométrica". Hilbert sostiene que la geometría es la "ciencia natural más completa", cuya diferencia fundamental respecto de otras teorías físicas reside únicamente en su avanzado estado de desarrollo. Empero esta posición empirista no es radicalizada exigiendo que todos los conceptos y leyes básicas de la geometría tengan un correlato empírico observable, sino que más bien se circunscribe a afirmar que esta teoría es, sólo en cuanto a su origen, una ciencia natural.

Podemos concluir que la imagen de la geometría y la concepción del método axiomático formal que presenta aquí Hilbert se oponen claramente a las posiciones radicalmente formalistas, con las que ha sido habitual identificarlo en las exposiciones de carácter más general. Poco tiene que ver el modo en que Hilbert entiende la naturaleza y la función del método axiomático formal, en particular en su aplicación a la geometría, con la concepción según la cual la matemática consiste en un sentido estricto en una colección de sistemas deductivos completamente formalizados, construidos a partir de un conjunto de principios o axiomas arbitrariamente escogidos y sin un significado intrínseco. Por el contrario, creo que he podido mostrar que un aspecto central de la temprana concepción axiomática de la geometría consiste en reconocer un papel relevante a las fuentes primarias de conocimiento geométrico - i.e. la experiencia y la intuición, en el proceso de axiomatización formal de la geometría elemental. En este respecto, considero que Hilbert conserva todavía una cierta actitud tradicional respecto de la naturaleza del conocimiento geométrico, que lo diferencia de otros partidarios de la concepción axiomática abstracta de la matemática surgida en las postrimerías del siglo XIX y en los inicios del siglo XX, como por ejemplo, Peano y Hausdorff.

Por último, aunque por lo general Hilbert se refiere a la "intuición geométrica o espacial" sin explicitar demasiado su significado y sin utilizar el término de un modo consistente, hemos identificado al menos dos sentidos diferentes en los que se alude a dicha noción. Por un lado, Hilbert concibe la intuición espacial en térmi- 
nos más bien empiristas, equiparándola con la percepción u observación de simples configuraciones de objetos en el espacio. Por otro lado, la "intuición geométrica" parece ser también entendida en un sentido más refinado, ligado a la práctica matemática, en donde es concebida como una cierta habilidad o capacidad, que puede ser instruida y desarrollada, para percibir relaciones geométricas fundamentales exhibidas generalmente en las construcciones geométricas o diagramáticas. La cuestión de la naturaleza y el estatus epistemológico de nuestra "intuición geométrica" es un problema sobre el que Hilbert no se pronuncia en estos cursos sobre geometría, en parte debido a que se trata de un problema filosófico que excede los límites de sus investigaciones axiomáticas de carácter puramente matemático. Y este hecho quizás se explica en virtud de que, como hemos defendido en este trabajo, en sus notas de clases Hilbert presenta la concepción axiomática de la geometría que subyace a su trabajo de carácter matemático en Fundamentos de la geometría, pero no elabora una filosofía de la geometría de manera sistemática. ${ }^{37}$

\section{Referencias bibliográficas}

Arana, A. y Mancosu, P. (2012): "On the Relationship between plane and solid Geometry”, The Review of Symbolic Logic, 5 (2), pp. 294-353.

Bernays, P. (1922a): "Die Bedeutung Hilberts für die Philosophie der Mathematik", Die Naturwissenschaften, 10, pp. 93-99.

Bernays, P. (1922b): "Über Hilberts Gedanken zur Grundlegung der Arithmetik", Jahresbericht der Deutschen Mathematiker-Vereinigung, 31, pp. 10-19.

CORry, L. (2004): David Hilbert and the Axiomatization of Physics (1898-1918): From 'Grundlagen der Geometrie' to 'Grundlagen der Physik', Dordrecht, Kluwer Academic Publishers.

Corry, L. (2006): "Axiomatics, Empirism, and Anschauung in Hilbert's Conception of Geometry: Between Arithmetic and General Relativity”, en J. Ferreirós y J. Gray (eds.), The Architecture of Modern Mathematics, Oxford University Press, Oxford, pp. 133-156.

Detlefsen, M. (1993): “Hilbert's Formalism”, Revue internationale de philosophie, 47, pp. 285-304.

Dieudonné, J. (1971): "Modern Axiomatic Method and the Foundations of Mathematics", en F. Le Lionnais (ed.), Great Currents of Mathematical Thought, vol. 2, New York, Dover Publications, pp. 251-266.

\footnotetext{
${ }^{37}$ La versión final de este trabajo se ha beneficiado substancialmente por los comentarios y sugerencias de los evaluadores de la Revista de Filosofía. El artículo ha sido realizado con el apoyo del CONICET (subsidio PIP 112-20110100-364).
} 


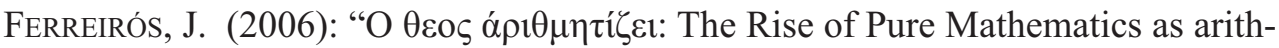
metic with Gauss", en C. Goldstein, N. Schappacher, J. Schwermer (eds.), The Shaping of Arithmetic: Number Theory after Carl Friedrich Gauss"s Disquistiones Arithmeticae, Berlin, Springer Verlag, pp. 235-268.

Ferreirós, J. (2009), "Hilbert, Logicism, and Mathematical Existence”, Synthese, 170, pp. 33-70.

FreGE, G. (1903): "Über die Grundlagen der Geometrie", Jahresbericht der Deutschen Mathematiker-Vereinigung, 12, pp. 319-324; pp. 368-375. Versión en español en Frege (1996), pp. 265-278.

FREGE, G. (1906): "Über die Grundlagen der Geometrie", Jahresbericht der Deutschen Mathematiker-Vereinigung, 15, pp. 293-309; pp. 377-404; pp. 423430. Versión en español en Frege (1996), pp. 279-334.

Frege, G. (1996): Escritos filosóficos, Barcelona, Crítica.

GAuss, C. y Bessel, F. (1880): Briefwechsel. Herausgegeben auf der königlich Preussischen Akademie der Wissenschaften, Leipzig, Engelman.

GRAY, J. (2007): Worlds Out of Nothing. A course in the History of Geometry in the 19th Century, London, Springer.

GiovanNini, E. (2012): “Una imagen de la realidad geométrica: la concepción axiomática de la geometría de Hilbert a la luz de la Bildtheorie de Heinrich Hertz", Crítica. Revista Hispanoamericana de Filosofia, 44, pp. 27-53.

Hallett, M. (2008): "Reflections on the Purity of Method in Hilbert's Grundlagen der Geometrie", en P. Mancosu (ed.), The Philosophy of Mathematical Practice, New York, Oxford, pp. 198-255.

HerzT, H. (1894): Gesammelte Werke, Band III: Die Prinzipien der Mechanik in neuem Zusammenhange dargestellt, Leipzig, Arthur Meiner.

Hilbert, D. (1891): Projektive Geometrie; (MS. Vorlesung, SS 1891). Niedersächsische Staats- und Universitätsbibliothek Göttingen, Handschriftenabteilung, Cod. Ms. D. Hilbert 535. Publicado parcialmente en Majer, U. \& Hallett, M. (2004), pp. 21-64.

HiLBerT, D. (1893/1894): Die Grundlagen der Geometrie; (MS. Vorlesung, WS 1893/4. Niedersächsische Staats- und Universitätsbibliothek Göttingen, Handschriftenabteilung, Cod. Ms. D. Hilbert 541. Publicado parcialmente en Majer, U. \& Hallett, M. (2004), pp. 72-178.

HiLberT, D. (1898): Mechanik; (MS. Vorlesung, WS 1898/9), Niedersächsische Staats- und Universitätsbibliothek Göttingen, Handschriftenabteilung, Cod. Ms. D. Hilbert 553.

HiLbert, D. (1896): "Über die Theorie der algebraischen Invarianten", en: Hilbert (1935), vol. 2, pp. 376-383.

HilBERT, D. (1898/1899a): Grundlagen der Euklidischen Geometrie, (MS. Vorlesung, WS 1898/9). Niedersächsische Staats- und Universitätsbibliothek 
Göttingen, Handschriftenabteilung, Cod. Ms. D. Hilbert 551. Publicado en U. MAJER \& M. HALLETT (2004), pp. 221-286.

HILBERT, D. (1898/1899b): Elemente der Euklidischen Geometrie, (MS. Vorlesung, WS 1898/9). Ausgearbeitet von Hans von Schaper. Niedersächsische Staatsund Universitätsbibliothek Göttingen, Handschriftenabteilung, Cod. Ms. D. Hilbert 552. Publicado en U. MAJER \& M. HALlett (2004), pp. 304-402.

HilberT, D. (1899): Grundlagen der Geometrie. Festschrift zur Feier der Enthüllung des Gauss-Weber Denkmals in Göttingen, Leipzig, Teubner. Reimpreso en U. MAJER \& M. HALLETT (2004).

HiLBERT, D. (1902): Grundlagen der Geometrie, (MS. Vorlesung, SS 1902). GeorgAugust-Universität Göttingen, Mathematisches Institut, Lesesaal. Publicado U. MAJER \& M. HALletT (2004), pp. 540-608.

HiLbert, D. (1900a): "Mathematische Probleme", en Hilbert (1935), pp. 290-329. Hilbert, D. (1905a): Logische Principien des mathematischen Denkens; (MS. Vorlesung, SS 1905). Ausgearbeitet von M. Born. Niedersächsische Staats- und Universitätsbibliothek Göttingen, Handschriftenabteilung, Cod. Ms. D. Hilbert 558a.

HilberT, D. (1905a): Logische Principien des mathematischen Denkens; (MS. Vorlesung, SS 1905). Ausgearbeitet von E. Hellinger. Georg-August-Universität Göttingen, Mathematisches Institut, Lesesaal.

Hilbert, D. (1918): “Axiomatisches Denken”, Mathematische Annalen, 78, pp. 405-415.

Hilbert, D. y Bernays, P. (1934): Grundlagen der Mathematik, vol. 1, Berlin, Springer Verlag.

HiLBERT, D. (1935): Gesammelte Abhandlungen, vol. 3, Berlin, Springer Verlag. HiLBERT, D. (1992): Natur und mathematisches Erkennen, Basel, Birkhäuser.

Hilbert, D. y Cohn-Vossen, S. (1996): Anschauuliche Geometrie, Berlin, Springer, $2^{\circ}$ edicion.

Lassalle Casanave, A. (1996): "Formalismo metodológico", Papeles Uruguayos de Filosofía, 1 (1), pp. 58.

Majer, U. y Hallett, M. (EDS.) (2004): David Hilbert's Lectures on the Foundations of Geometry, 1891-1902, Berlin, Springer Verlag.

MAJER, U. (2006): "The Relation of Logic and Intuition in Kant's Philosophy of Science, Particularly Geometry", en Carson, E. y Huber, R.(Eds.), Intuition and the Axiomatic Method, Kluwer, Dordrecht, pp. 47-66.

Mancosu, P. (2010): The Adventure of Reason, New York, Oxford University Press. PASCH, M. (1882): Vorlesungen über neuere Geometrie, Leipzig, Teubner.

Poincaré, H. (1902): "[Review]: Hilbert. Les Foundaments de la Géometrie", Bulletin des sciences mathemátiques. Deuxième série, 26, pp. 249-272.

REID, C. (1996): Hilbert, Berlin, Springer. 
Rowe, D. (2000): "The Calm before the Storm: Hilbert's Early Views on the Foundations", en Hendriks, V. et al. (eds.), Proof Theory. History and Philosophical Significance, Dordrecht, Kluwer Academic Publishers, pp. 5593.

Schlimm, D. (2010): "Pasch's Philosophy of Mathematics", The Review of Symbolic Logic, 3 (1), pp. 1-26.

SMADJA, I. (2012): "Local axioms in disguise: Hilbert on Minkowski diagrams", Synthese, 186, pp. 315-370.

Toepell, M. (1986): Über die Entstehung von David Hilberts Grundlagen der Geometrie, Göttingen, Vandenhoeck \& Ruprecht.

Torres Alcaraz, C. (2009): "De la matemática clásica a la matemática moderna: Hilbert y el esquematismo kantiano", Diánoia, LIV (63), pp. 37-70.

Torretti, R. (1984): Philosophy of Geometry from Riemann to Poincaré, Dordrecht, Kluwer, $2^{\circ}$ edición.

Weyl, H. (1925): "Die heutige Erkenntnislage in der Mathematik", Symposion, 1, pp. 1-32.

Eduardo N. Giovannini

Consejo Nacional de Investigaciones

Científicas y Técnicas (CONICET), Argentina

engiovannini@conicet.gov.ar 